\section{Criterios para la indicación selectiva de glucocorticoides en pacientes con tumores hipofisiarios sometidos a cirugía transesfenoidal}

\author{
CARMEN A. CARRASCO ${ }^{1}$, PABLO VILLANUEVA G. ${ }^{2}$
}

\author{
'Departamento de \\ Endocrinología. \\ ${ }^{2}$ Departamento de Neurocirugía. \\ Facultad de Medicina, Pontifica \\ Universidad Católica de Chile; \\ Santiago de Chile. \\ Recibido el 30 de junio de 2014, \\ aceptado el 14 de agosto de \\ 2014.
Correspondencia a: Carmen A. Carrasco Dirección: Lira 85 piso 5; Santiago de Chile. Fono: (562)3543095 cantonie@med.puc.cl

Background: There is consensus in promoting the selective use of glucocorticoids (GC) in the peri-operative period of transsphenoidal surgery (TE) for pituitary adenomas (PA). Aim: To evaluate the safety of a selective glucocorticoid administration protocol and the usefulness of immediate postoperative cortisol levels as a predictor of final eucortisolism. Patients and Methods: Clinical and biochemical data from 40 patients aged 27 to 78 years (65\% males) were prospectively collected. Exclusion criteria were previous use of GC, apoplexy and Cushing disease. Patients with pre-operative short synthetic ACTH test (SST) $>18 \mu \mathrm{g} / \mathrm{dl}$ or basal cortisol $>15 \mu \mathrm{g} / \mathrm{dl}$ did not receive GC. A morning serum cortisol (SC) threshold of $10 \mu \mathrm{g} / \mathrm{dl}$ in postoperative days one to three was used to decide a discharge without GC. Hypotension, dizziness or nausea, requirement of increased dose of corticosteroids, hospitalizations and emergency service visits were investigated, as well as surgical and endocrinological complications. Corticotropic status was evaluated three months after surgery. Results: Macroadenomas were present in $87 \%$ of patients. Median hospital stay was 4 days and follow up lasted 9 months. No differences were found in gender, age or tumor size between patients who received or not GC (35 and 65\% respectively). Eighty five percent of patients were discharged without GC and all of them had normal corticotropic function three months after surgery. A SC $\geq 15 \mu \mathrm{g} / \mathrm{dl}$ had 100\% specificity to predict eucortisolism. Conclusions: Selective glucocorticoid administration is safe. A normal corticotropic function before surgery and in the immediate postoperative period are useful to identify patients who do not need GC.

(Rev Med Chile 2014; 142: 1113-1119) plasms.

Key words: Glucocorticoids; Perioperative procedures; Pituitary neo-

U na de las complicaciones de la cirugía transesfenoidal de los adenomas hipofisiarios es el hipocortisolismo post-operatorio, el cual se produce por resección de la hipófisis normal durante el acto quirúrgico. Esta complicación está determinada por le experiencia del neurocirujano, el número de cirugías previas y el tamaño tumoral ${ }^{1}$. La incidencia reportada es variable, con cifras que van desde $1 \%$ hasta $10,3 \%{ }^{2-5}$. En el caso de resección de macroadenomas gigantes (mayores de $3 \mathrm{~cm}$ ), el compromiso de más de un eje hipofisiario producto del acto quirúrgico puede alcanzar hasta $13 \%{ }^{1}$. En algunos centros, el temor a una crisis adrenal ha motivado la administración 
rutinaria de glucocorticoides peri-operatorios, pero la conducta sigue siendo heterogénea ${ }^{6,7}$. Por otra parte, el uso de glucocorticoides no está exento de riesgos ya que puede enmascarar una diabetes insípida post-operatoria en forma dosis dependiente ${ }^{8}$ y dificultar la evaluación del estatus definitivo del eje corticotrópico ${ }^{9}$. A su vez, la falta de evaluación del eje corticotrópico en el post operatorio inmediato promueve la indicación de corticoides al alta, con dosis que pueden ser supra fisiológicas ${ }^{7}$, exponiendo a los pacientes a complicaciones crónicas como supresión adrenal, osteoporosis, hiperglicemia y aumento de la presión intraocular ${ }^{10-11}$. Según el Departamento de Información y Estadísticas de Salud DEIS, del MINSAL, hubo 246 egresos por adenomas hipofisiarios en Chile durante el 2010 y se estima que la incidencia llegaría a 1.39 por 100.000 habitantes para adenoma hipofisiario.

Considerando la baja incidencia de hipocortisolismo en pacientes sometidos a cirugía transesfenoidal y los efectos secundarios de los glucocorticoides, algunos expertos han promovido la administración selectiva de glucocorticoides en el post-operatorio. Las guías clínicas publicadas en el 2002 proponen no administrar glucocorticoides en pacientes con un eje corticotrópico preoperatorio normal ${ }^{12}$. La necesidad de corticoides al alta dependería a su vez del cortisol plasmático postoperatorio inmediato. Los autores de estas guías clínicas enfatizan la utilidad de contar con más estudios prospectivos que avalen estas sugerencias.

Nuestros objetivos fueron evaluar la seguridad de un protocolo de administración selectiva de glucocorticoides y la utilidad del cortisol post operatorio inmediato como predictor de eucortisolismo post quirúrgico.

\section{Pacientes y Método}

\section{Pacientes}

Se incluyeron todos los pacientes mayores de 18 años con diagnóstico de adenoma hipofisiario sometidos a resección transesfenoidal por el mismo neurocirujano (PV) entre marzo de 2008 y diciembre de 2011. Se excluyeron los pacientes con diagnóstico de Enfermedad de Cushing $(\mathrm{n}=2)$, apoplejía hipofisiaria $(\mathrm{n}=1)$ y aquellos en tratamiento glucocorticoideo previo al reclutamiento $(\mathrm{n}=4)$.
Se registró el tamaño tumoral en todos los pacientes, considerando el diámetro mayor medido en la imagen de la resonancia magnética. De acuerdo al tamaño, los tumores se clasificaron en microadenomas $(\leq 10 \mathrm{~mm})$ y macroadenomas $(>10 \mathrm{~mm})$. Adicionalmente, se registró la histología y secreción hormonal de los tumores incluidos.

\section{Protocolo de administración de glucocorticoides}

Se consideró normal un cortisol plasmático $\geq 18 \mu \mathrm{g} / \mathrm{dl}$ a los $60 \mathrm{~min}$ post-estimulación con 250 $\mu \mathrm{g}$ de ACTH sintético (Synacthen ${ }^{\circledR}$, Novartis). En 22 pacientes, sólo se contó con un cortisol plasmático basal, medido en ayunas antes de las 9:00 am y se consideró normal un cortisol plasmático basal $\geq 15 \mu \mathrm{g} / \mathrm{dl}^{2,13-16}$.

Los pacientes fueron clasificados en dos grupos según los siguientes criterios.

Grupo 1: cortisol plasmático post-estímulo $\geq 18 \mu \mathrm{g} / \mathrm{dl}$ o basal $\geq 15 \mu \mathrm{g} / \mathrm{dl}$.

Grupo 2: cortisol plasmático post-estímulo $<18 \mu \mathrm{g} / \mathrm{dl}$ o basal $<15 \mu \mathrm{g} / \mathrm{dl}$.

Los pacientes del Grupo 1 no recibieron corticoides y se controlaron con cortisol post operatorio a las $24 \mathrm{~h}$ post cirugía. Los pacientes del Grupo 2 recibieron $50 \mathrm{mg}$ de hidrocortisona cada $8 \mathrm{~h}$ por vía intravenosa el día de la cirugía (día 0), $20 \mathrm{mg}$ cada 12 h por vía oral (vo) el día 1, y $20 \mathrm{mg}$ vo a las 07:00 AM el día 2. El día 3 se midió cortisol plasmático basal antes de las 9:00 am.

Los pacientes con cortisol post-operatorio $\geq 15 \mu \mathrm{g} / \mathrm{dl}$ fueron dados de alta sin reemplazo glucocorticoideo. Los pacientes con cortisol entre 10 y $14,9 \mu \mathrm{g} / \mathrm{dl}$ fueron dados de alta sin reemplazo glucocorticoideo, pero fueron educados para recibir hidrocortisona en caso de estrés. Los pacientes con cortisol plasmático post-operatorio $<10 \mu \mathrm{g} / \mathrm{dl}$ fueron dados de alta con $15 \mathrm{mg}$ de hidrocortisona vía oral, fraccionados en dosis de $10 \mathrm{mg}$ a las 8:00 am y $5 \mathrm{mg}$ a las 5:00 pm (Figura 1).

La utilidad del cortisol post operatorio como predictor del status corticotrópico definitivo se realizó a los tres meses post cirugía mediante la medición de un cortisol plasmático basal a las 8:00 am y/o una hora post-estimulación con ACTH 250 $\mu \mathrm{g}$ (Synacthen $\left.{ }^{\circledR}\right)$. Se consideraron normales un cortisol post estimulo $\geq 18 \mu \mathrm{g} / \mathrm{dl}$ o basal $\geq 15 \mu \mathrm{g} / \mathrm{dl}$.

\section{Seguridad del protocolo}

Durante la hospitalización se registraron eventuales episodios de hipotensión, síntomas de 


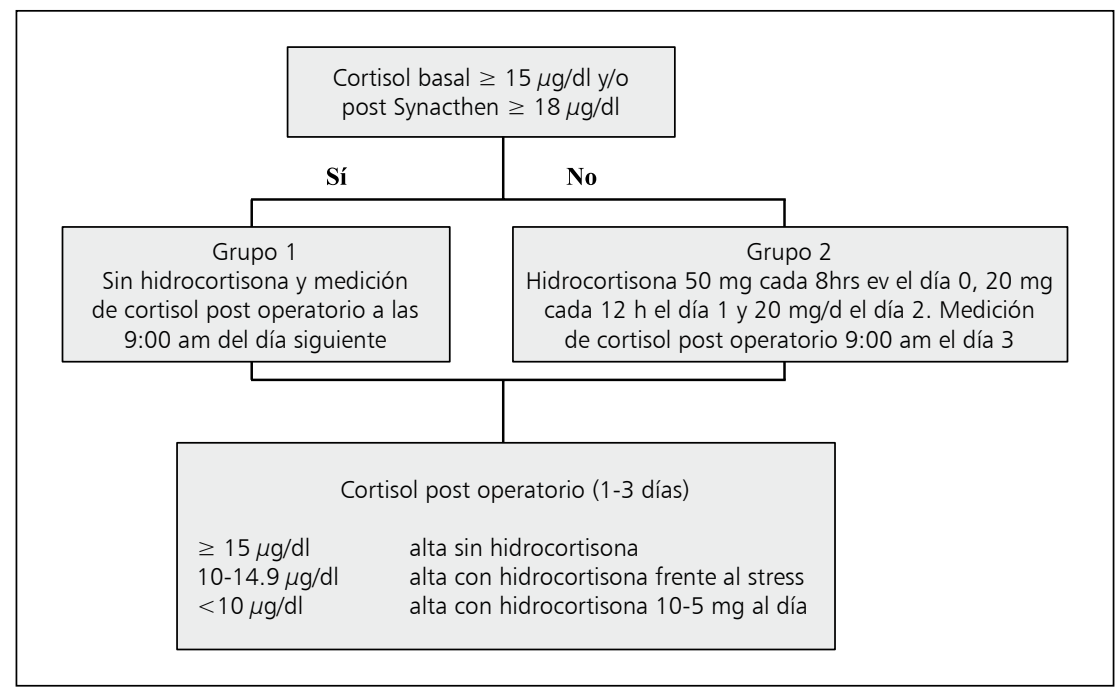

Figura 1. Protocolo de administración selectiva de glucocorticoides para cirugía transesfenoidal de tumores selares. hipocortisolismo como mareos o náuseas, necesidad de uso o aumento de dosis de corticoides. Tres meses post cirugía se investigó la ocurrencia de hospitalizaciones y consultas al servicio de urgencia, necesidad de aumento o uso de corticoides y síntomas de hipocortisolismo.

Durante la hospitalización se registró la presencia de complicaciones tanto endocrinológicas como quirúrgicas. Se midió diuresis cada 6 h durante las primeras $24 \mathrm{~h}$ post-cirugía, y luego cada $24 \mathrm{~h}$. Se controló sodio plasmático diariamente hasta el alta. Se definió como diabetes insípida (DI) la presencia de diuresis mayor a 1 litro en $6 \mathrm{~h}$ o 4 litros en $24 \mathrm{~h}$, en presencia de sodio plasmático $\geq 145 \mathrm{mEq} / \mathrm{L}$ y glicemia normal ${ }^{17}$. Se definió como síndrome de secreción inapropiada de hormona anti-diurética (SIADH) la presencia de sodio plasmático $<135 \mathrm{mEq} / \mathrm{L}$ en el post operatorio, en ausencia de administración de líquidos parenterales, hipotiroidismo, hipocortisolismo o uso de diuréticos ${ }^{18}$. Todos los pacientes fueron controlados a los 7 días post-alta con sodio plasmático y evaluación clínica para detectar la presencia de poliuria, nicturia y polidipsia.

Este estudio fue aprobado por el Comité de ética del Hospital Clínico de la Pontificia Universidad Católica de Chile.

\section{Estudios de laboratorio}

Se midió cortisol sérico con inmunoensayo de electroquimioluminiscencia automático (Roche Diagnostics GmbH, Mannheim, Alemania) siguiendo las especificaciones del fabricante. El coeficiente de variación analítico inter ensayo es de $1,7 \%$ para concentraciones de $14,8 \mu \mathrm{g} / \mathrm{dL}$ y $1,4 \%$ para concentraciones de $30,7 \mu \mathrm{g} / \mathrm{dl}$, con una sensibilidad analítica de $0,018 \mu \mathrm{g} / \mathrm{dL}$.

El test de estimulación se realizó mediante la administración de $250 \mu \mathrm{g}$ de ACTH sintético (Synacthen) y midiendo cortisol plasmático a los $60 \mathrm{~min}$. El cortisol $(\mu \mathrm{g} / \mathrm{dl})$ puede ser convertido a SI units (nmol/l) multiplicando por 27,59.

\section{Estadística}

Los valores de cortisol plasmático pre y postoperatorio se expresan como mediana y dispersión. La comparación de los valores de cortisol plasmático post-operatorio entre los grupos 1 y 2 se realizó mediante pruebas no paramétricas para variables independientes (Mann-Whitney). Para la comparación entre variables categóricas se utilizó el test exacto de Fisher. Se consideró estadísticamente significativo un valor $\mathrm{p}<0,05$. Para el análisis estadístico se utilizó el programa SPSS para Windows versión 15.0 (SPSS, Inc, Chicago, IL).

\section{Resultados}

\section{Características clínicas y bioquímicas de los pacientes incluidos}

Se incluyeron 40 pacientes, 16 mujeres y 24 hombres, con una edad mediana de 45 años (dispersión 27 a 78). El 87\% $(\mathrm{n}=35)$ de los tu- 
Tabla 1. Características clínicas de los pacientes incluidos según función corticotrópica pre operatoria

\begin{tabular}{|lccc|}
\hline & Grupo 1 (n = 26) & Grupo 2 (n = 14) & p \\
\hline Edad, mediana en años (dispersión) & $45(29-78)$ & $45(27-76)$ & NS \\
Género (Mujer/Hombre) & $8 / 18$ & $8 / 6$ & NS \\
Tamaño: micro/macro & $4 / 22$ & $1 / 13$ & NS \\
Tamaño en mm & $24(6-40)$ & $22(9-29)$ & NS \\
Días de hospitalización mediana (dispersión) & $4(3-8)$ & $4(3-8)$ & NS \\
Cortisol basal pre op $(\mu \mathrm{g} / \mathrm{dl})$ & $12,3(6,5-35)$ & $9,9(3,3-14,8)$ & 0,028 \\
Cortisol post op inmediato $(\mu \mathrm{g} / \mathrm{dl})$ & $24,6(7,6-50)$ & $20,7(2.2-34,6)$ & $\mathrm{NS}$ \\
Seguimiento & $6(3-36)$ & $13(6-34)$ & $\mathrm{NS}$ \\
\hline
\end{tabular}

mores eran macroadenomas. Del total de tumores incluidos, $57 \%(\mathrm{n}=23)$ correspondían a adenomas gonadotropos no secretores, $32 \%(n=13)$ a adenomas somatotropos, dos nulls cells y dos corticotropos silentes. El cortisol plasmático basal pre-operatorio de todo el grupo fue de $12 \mu \mathrm{g} / \mathrm{dl}$ (dispersión 3,3-35).

Un total de 26 pacientes fueron clasificados en el grupo 1 y 14 en el grupo 2.

La Tabla 1 muestra que no hubo diferencias de edad, género, tamaño tumoral ni tiempo de hospitalización entre ambos grupos. El cortisol plasmático basal pre operatorio fue mayor en el Grupo 1 (p 0,028). Un total de 7 tumores eran mayores de $3 \mathrm{~cm}$ (rango 3-4,4 cm), todos con eje corticotrópico conservado en la evaluación pre operatoria.

El 50\% de los pacientes presentaba compromiso de algún eje pituitario, siendo el compromiso del eje gonadal el más frecuente (42\%).

\section{Seguridad de la aplicación del protocolo}

Ningún paciente presentó hipotensión o síntomas sugerentes de crisis adrenal. Los pacientes estaban asintomáticos a los tres meses post cirugía y sin registro de eventos sugerentes de insuficiencia corticotropica. La mediana de hospitalización fue de 4 días (dispersión 3-8) y el seguimiento de 10 meses (dispersión 3-36).

El 17\% de los pacientes presentó DI post-operatoria transitoria y $2 \%$ definitiva (un paciente). El 7\% presentó SIADH que remitió dentro de la semana de la cirugía. Un paciente evolucionó con fístula de líquido cefalorraquídeo que se manejó con drenaje epidural y resolvió a los 3 días de la cirugía. La tasa de estas complicaciones no difirió entre los grupos 1 y 2.

\section{Cortisol post-operatorio en Grupos 1 y 2}

No hubo diferencias entre la cortisol post operatorio del Grupo $1(24,6 \mu \mathrm{g} / \mathrm{dl}$ [dispersión 7,3-50]); y el grupo $2(20,7 \mu \mathrm{g} / \mathrm{dl}$ [dispersión 2,2$34,6]$ (p 0,2) (Figura 2).

El $85 \%$ de los pacientes tenían cortisol a las $24 \mathrm{~h}>10 \mu \mathrm{g} / \mathrm{dl}$ y fueron dados de alta sin terapia glucocorticoidea.

\section{Cortisol post-operatorio como predictor de la función corticotropa post operatoria}

Todos los pacientes estaban eucortisólicos a los tres meses post cirugía, incluyendo los 7 pacientes con macroadenomas gigantes.

Llama la atención que un paciente con cortisol post operatorio de $2,2 \mu \mathrm{g} / \mathrm{dl}$ tenía función corticotrópica normal a los tres meses, con cortisol post Synacthen de 27,8 $\mu \mathrm{g} / \mathrm{dl}$. Retrospectivamente se comprobó que había recibido dexametasona durante la inducción de la anestesia, lo cual pensamos podría explicar este hallazgo. Este paciente fue excluido del análisis.

Un valor de cortisol plasmático post-operatorio $\geq 15 \mu \mathrm{g} / \mathrm{dl}$ tuvo una especificidad de $72,5 \%$ para identificar los eucortisolicos y un valor predictivo negativo de $100 \%$ para descartar hipocortisolismo. Un valor de cortisol plasmático post-operatorio $\geq 10 \mu \mathrm{g} / \mathrm{dl}$ tuvo el mismo valor predictivo negativo y una especificidad de $87,2 \%$.

La ausencia de insuficiencia corticotrópica post operatoria impidió el cálculo de sensibilidad y valor predictivo positivo. 


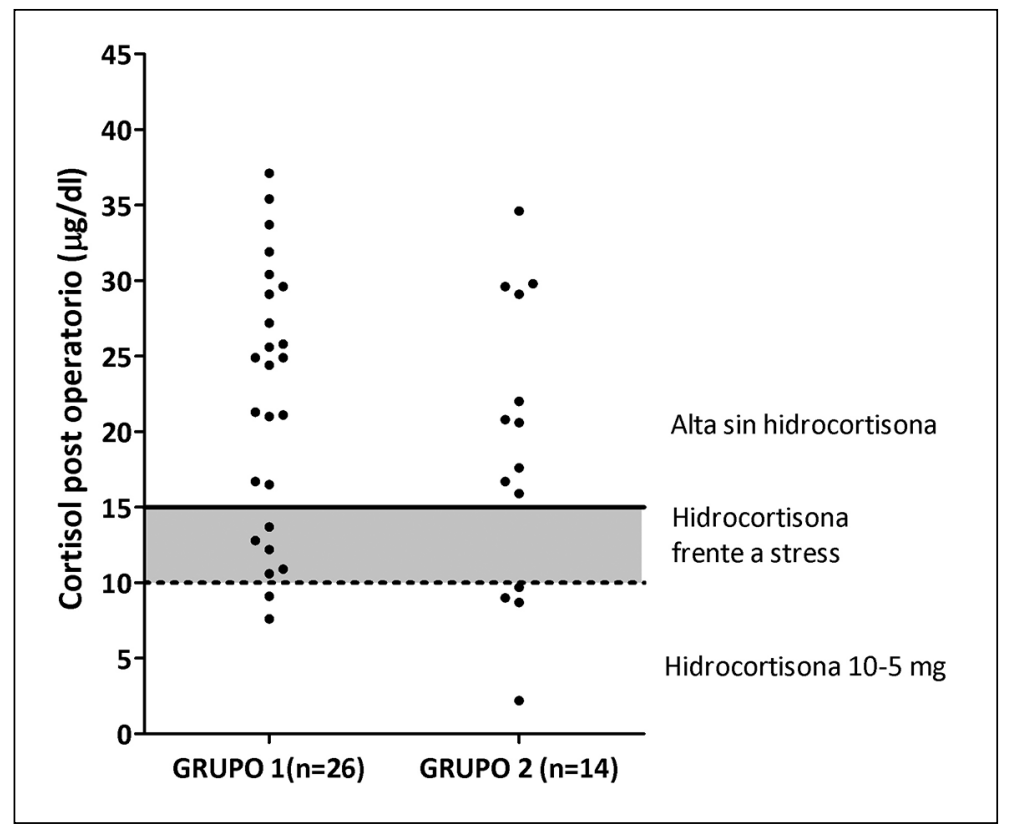

Figura 2. Cortisol post operatorio (1-3 días) según cortisol máximo pre operatorio. Se incluye la indicación de corticoides al alta según protocolo.

\section{Discusión}

Nuestros resultados muestran que el uso selectivo y protocolizado de glucocorticoides perioperatorios es seguro. Por otra parte, el cortisol post operatorio inmediato permite seleccionar a los pacientes que no requieren corticoides al alta.

\section{Seguridad de protocolos de administración selectiva}

La aplicación de este protocolo en nuestra población resultó segura, sin eventos agudos sugerentes de insuficiencia suprarrenal ni consultas al servicio de urgencia.

Una ventaja de nuestro protocolo es que permite la aleatorización de pacientes en base a un cortisol basal en el caso de no contar con un test de Synacthen. Un meta análisis de 13 estudios sugirió que un cortisol basal superior a $365 \mathrm{nmol} / \mathrm{L}$ $(13 \mu \mathrm{g} / \mathrm{dl})$ permitía excluir una insuficiencia corticotrópica en pacientes en estudio por patología hipofisiaria ${ }^{16}$. En un estudio más reciente, Yip et $\mathrm{al}^{19}$ apoyan estos datos con un $100 \%$ de valor predictivo positivo en pacientes con cortisol basal superior a $266 \mathrm{nmol} / \mathrm{l}(10 \mu \mathrm{g} / \mathrm{dl})$. A nivel local, Díaz et al encontraron resultados similares, con $100 \%$ de eucortisolismo al tener un cortisol basal $>10 \mu \mathrm{g} / \mathrm{dl}^{20}$.
Nosotros decidimos adoptar una conducta más conservadora y considerar como eucortisolicos a los pacientes con un cortisol basal $>15 \mu \mathrm{g} / \mathrm{dl}^{2,13-16}$.

Respecto a la ausencia de hipocortisolismo post operatorio, nuestra población incluyó $17 \%$ de macroadenomas mayores de $3 \mathrm{~cm}$, con función cortisolica pre operatoria conservada. La baja frecuencia de macroadenomas gigantes puede explicar la ausencia de hipocortisolismo post operatorio. No contamos con análisis volumétrico tumoral pre y post operatorio que nos permita evaluar el grado de resección tumoral, el cual también es un factor que podría influir en la presencia o no de hipocortisolismo post operatorio.

\section{Pacientes que pueden ser incluidos en estos protocolos}

Nuestro protocolo excluye aquellos pacientes con uso crónico de corticoides, apoplejía y cirugía transcraneana. Esto es una conducta que se repite en trabajos previos y no existen evidencias que avalen la seguridad de este protocolo en pacientes con esas características.

\section{Valor predictivo de eucortisolismo del cortisol post-operatorio inmediato}

El nivel de corte del cortisol plasmático postoperatorio como predictor de eucortisolismo es 
variable según los estudios ${ }^{5,21-27}$. Un meta-análisis concluyó que valores bajo $5 \mu \mathrm{g} / \mathrm{dl}$ y superiores a $13 \mu \mathrm{g} / \mathrm{dl}$ son altamente predictores de deficiencia e indemnidad del eje corticotrópico, respectivamente $^{15}$, cifras inferiores a las propuestas en las guías clínicas ${ }^{12}$. Esto puede explicar el porqué pacientes catalogados como hipocortisólicos en el pre operatorio inmediato y que fueron dados de alta con hidrocortisona, estaban eucortisólicos en la evaluación de los 3 meses.

Nuestro estudio concuerda con lo planteado por otros autores en el sentido de que valores sobre $15 \mu \mathrm{g} / \mathrm{dl}$ permiten predecir con alta seguridad la preservación del eje corticotropico post cirugía $a^{5,12,21,28}$. Si bien encontramos que pacientes con cortisol de 10 a $14,9 \mu \mathrm{g} / \mathrm{dl}$ también muestran preservación del eje, no es posible sacar conclusiones definitivas debido al número limitado de pacientes en este grupo $(n=5)$. Una alternativa para evitar sobre tratar a estos pacientes sería evaluar con un nuevo cortisol basal antes de los tres meses, pero queda aún por definir si un cortisol bajo $10 \mu \mathrm{g} / \mathrm{dl}$ es suficientemente seguro.

El valor predictivo del cortisol post operatorio inmediato disminuye frente al uso de corticoides empíricos en el peri operatorio. Un trabajo retrospectivo en el cual se indicó en forma rutinaria corticoides mostró una especificidad de 69,2\% para predecir eucortisolismo ${ }^{26}$, cifra inferior a nuestros resultados y a lo publicado en estudios prospectivos $^{5}$. Esto podría explicarse por la supresión de los corticotropos normales frente a los corticoides exógenos y la dificultad en la recuperación espontánea del eje adrenal.

Nuestro trabajo tiene la limitante de que no todos los pacientes fueron sometidos a un test de synacthen y es posible que los pacientes con un cortisol basal $<15 \mu \mathrm{g} / \mathrm{dl}$ hubiesen respondido correctamente al test de estimulación. Sin embargo, esto no invalida nuestros resultados respecto a la seguridad del protocolo en la identificación de pacientes que pueden ser dados de alta sin corticoides $\mathrm{ni}$ al valor predictor del cortisol post operatorio. La ausencia de hipocortisolismo post quirúrgico nos impidió además el cálculo de los falsos negativos del cortisol post operatorio, reportado en el 1 a $4,7 \%$ de los casos en estudios previos ${ }^{5,21,28}$.

En conclusión, la aplicación de un protocolo de administración selectiva de glucocorticoides en el peri-operatorio de la cirugía transesfenoidal de adenomas hipofisiarios es segura. Su implemen- tación permite seleccionar los pacientes que no requieren corticoides durante y/o después de la cirugía y evitar la administración iatrogénica de la terapia esteroidal.

\section{Referencias}

1. Hofstetter C, Nanaszko M, Mubita L, Tsiouris J, Anand $\mathrm{V}$, Schwartz T. Volumetric classification of pituitary macroadenomas predicts outcome and morbidity following endoscopic endonasal transsphenoidal surgery. Pituitary 2012; 15: 450-63.

2. Cozzi R, Lasio G, Cardia A, Felisati G, Montini M, Attanasio R. Perioperative cortisol can predict hypothalamus-pituitary-adrenal status in clinically nonfunctioning pituitary adenomas. J Endocrinol Invest 2009; 32: 460-4.

3. Nomikos P, Ladar C, Fahlbusch R, Buchfelder M. Impact of primary surgery on pituitary function in patients with non-functioning pituitary adenomas. A study on 721 patients. Acta Neurochir (Wien) 2004; 146: 27-35.

4. Kristof RA, Wichers M, Haun D, Redel L, Klingmuller D, Schramm J. Peri-operative glucocorticoid replacement therapy in transsphenoidal pituitary adenoma surgery: a prospective controlled study. Acta Neurochir (Wien) 2008; 150: 329-35.

5. Marko NF, Hamrahian AH, Weil RJ. Immediate postoperative cortisol levels accurately predict postoperative hypothalamic-pituitary-adrenal axis function after transsphenoidal surgery for pituitary tumors. Pituitary 2010; 13: 249-55.

6. Nemergut EC, Dumont AS, Barry UT, Laws ER. Perioperative management of patients undergoing transsphenoidal pituitary surgery. Anesth Analg 2005; 101: 1170-81.

7. Reynolds RM, Stewart PM, Seckl JR, Padfield PL. Assessing the HPA axis in patients with pituitary disease: a UK survey. Clin Endocrinol (Oxf) 2006; 64: 82-5.

8. Rajaratnam S, Seshadri MS, Chandy MJ, Rajshekhar V. Hydrocortisone dose and postoperative diabetes insipidus in patients undergoing transsphenoidal pituitary surgery: a prospective randomized controlled study. $\mathrm{Br}$ J Neurosurg 2003; 17: 437-42.

9. Klose M, Lange M, Kosteljanetz M, Poulsgaard L, Feldt-Rasmussen U. Adrenocortical insufficiency after pituitary surgery: an audit of the reliability of the conventional short synacthen test. Clin Endocrinol (Oxf) 2005; 63: 499-505.

10. Agha A, Liew A, Finucane F, Baker L, O’Kelly P, Tormey W, Thompson CJ. Conventional glucocorticoid replace- 
ment overtreats adult hypopituitary patients with partial ACTH deficiency. Clin Endocrinol (Oxf) 2004; 60: 68893.

11. Peacey SR, Guo CY, Robinson AM, Price A, Giles MA, Eastell R, et al. Glucocorticoid replacement therapy: are patients over treated and does it matter? Clin Endocrinol (Oxf) 1997; 46: 255-61.

12. Inder WJ, Hunt PJ. Glucocorticoid replacement in pituitary surgery: guidelines for perioperative assessment and management. J Clin Endocrinol Metab 2002; 87: 2745-50.

13. Pereira O, Bevan JS. Preoperative assessment for pituitary surgery. Pituitary 2008; 11:347-51.

14. Deutschbein T, Unger N, Mann K, Petersenn S. Diagnosis of secondary adrenal insufficiency in patients with hypothalamic-pituitary disease: comparison between serum and salivary cortisol during the high-dose short synacthen test. Eur J Endocrinol 2009; 160: 9-16.

15. Hagg E, Asplund K, Lithner F. Value of basal plasma cortisol assays in the assessment of pituitary-adrenal insufficiency. Clin Endocrinol (Oxf) 1987; 26: 221-6.

16. Kazlauskaite R, Evans AT, Villabona CV, Abdu TA, Ambrosi B, Atkinson AB, et al. Corticotropin tests for hypothalamic-pituitary-adrenal insufficiency: a metaanalysis. J Clin Endocrinol Metab 2008; 93: 4245-53.

17. Olson BR, Gumowski J, Rubino D, Oldfield EH. Pathophysiology of hyponatremia after transsphenoidal pituitary surgery. J Neurosurg 1997; 87: 499-507.

18. Loh JA, Verbalis JG. Disorders of water and salt metabolism associated with pituitary disease. Endocrinol Metab Clin North Am 2008; 37: 213-34.

19. Yip CE, Stewart SA, Imran F, Clarke DB, Mokashi A, Kaiser SM, et al. The role of morning basal serum cortisol in assessment of hypothalamic pituitary-adrenal axis. Clin Invest Med 2013; 36: E216-222.

20. Díaz E, Ladrón de Guevara A, García M, Wohllk N. Cortisol plasmático basal matinal como predictor de indemnidad del eje Hipotálamo Hipófisis Suprarrenal. Rev Chil Endocrinol diabetes 2008; 4: 234-37.

21. Wentworth JM, Gao N, Sumithran KP, Maartens NF, Kaye AH, Colman PG, et al. Prospective evaluation of a protocol for reduced glucocorticoid replacement in transsphenoidal pituitary adenomectomy: prophylactic glucocorticoid replacement is seldom necessary. Clin Endocrinol (Oxf) 2008; 68: 29-35.

22. Courtney CH, McAllister AS, McCance DR, Bell PM, Hadden DR, Leslie H, et al. Comparison of one week $0900 \mathrm{~h}$ serum cortisol, low and standard dose synacthen tests with a 4 to 6 week insulin hypoglycaemia test after pituitary surgery in assessing HPA axis. Clin Endocrinol (Oxf) 2000; 53: 431-6.

23. Courtney $\mathrm{CH}$, McAllister AS, McCance DR, Hadden DR, Leslie H, Sheridan B, et al. The insulin hypoglycaemia and overnight metyrapone tests in the assessment of the hypothalamic-pituitary-adrenal axis following pituitary surgery. Clin Endocrinol (Oxf) 2000; 53: 30912.

24. Hout WM, Arafah BM, Salazar R, Selman W. Evaluation of the hypothalamic-pituitary-adrenal axis immediately after pituitary adenomectomy: is perioperative steroid therapy necessary? J Clin Endocrinol Metab 1988; 66: 1208-12.

25. Auchus RJ, Shewbridge RK, Shepherd MD. Which patients benefit from provocative adrenal testing after transsphenoidal pituitary surgery? Clin Endocrinol (Oxf) 1997; 46: 21-7.

26. Jayasena CN, Gadhvi KA, Gohel B, Martin NM, Mendoza N, Meeran K, et al. Day 5 morning serum cortisol predicts hypothalamic-pituitary-adrenal function after transsphenoidal surgery for pituitary tumors. Clin Chem 2009; 55: 972-7.

27. Karaca Z, Tanriverdi F, Atmaca H, Gokce C, Elbuken G, Selcuklu A, et al. Can basal cortisol measurement be an alternative to the insulin tolerance test in the assessment of the hypothalamic-pituitary-adrenal axis before and after pituitary surgery? Eur J Endocrinol 2010; 163: 37782.

28. Marko NF, Gonugunta VA, Hamrahian AH, Usmani A, Mayberg MR, Weil RJ. Use of morning serum cortisol level after transsphenoidal resection of pituitary adenoma to predict the need for long-term glucocorticoid supplementation. J Neurosurg 2009; 111: 540-4. 